\title{
A stable interpenetrated Zn-MOF with efficient light hydrocarbon adsorption/separation performance
}

Xia Wang ${ }^{\ddagger}$, Xiaokang Wang ${ }^{\ddagger}$, Xiurong Zhang, Weidong Fan, Qing Li, Weifeng Jiang, Fangna Dai*, Daofeng Sun

College of Science, School of Materials Science and Engineering, China University of Petroleum (East China), Qingdao, Shandong 266580, People's Republic of China.

* Fangna Dai Email: fndai@upc.edu.cn. 


\section{Contents}

Section 1. Experimental Section

Section 2. Crystal data, structure and characterization of UPC-98

Section 3. Simulation and calculation for gas selectivity by IAST methods

Section 4. Simulation and calculation for $Q_{\text {st }}$ by Clausius-Clapeyron equation 


\section{Section 1. Experimental Section}

\subsection{Materials and characterizations.}

All chemical reagents used were available from chemical suppliers and were not purified. The powder XRD diffractograms (PXRD) were acquired on the Panalytical X-Pert PRO diffractometer $(\mathrm{Cu}-\mathrm{K} \alpha)$. Thermogravimetric analysis (TGA) measurement was conducted on the Mettler Toledo TGA instrument under the $\mathrm{N}_{2}$ atmosphere within the $40-900{ }^{\circ} \mathrm{C}$ region with a heating speed of $10{ }^{\circ} \mathrm{C} / \mathrm{min}$. Infrared (IR) spectrum was obtained on the Nicolet 330 FTIR Spectrometer within the range of $4000-400 \mathrm{~cm}^{-1}$. Elementary analyses $(\mathrm{C}, \mathrm{H}, \mathrm{N})$ were performed on the $\mathrm{CE}$ instruments EA 1110 elemental analyzer.

\subsection{Synthesis of $\mathrm{H}_{3} \mathrm{BTB}-\mathrm{NH}_{2}$.}

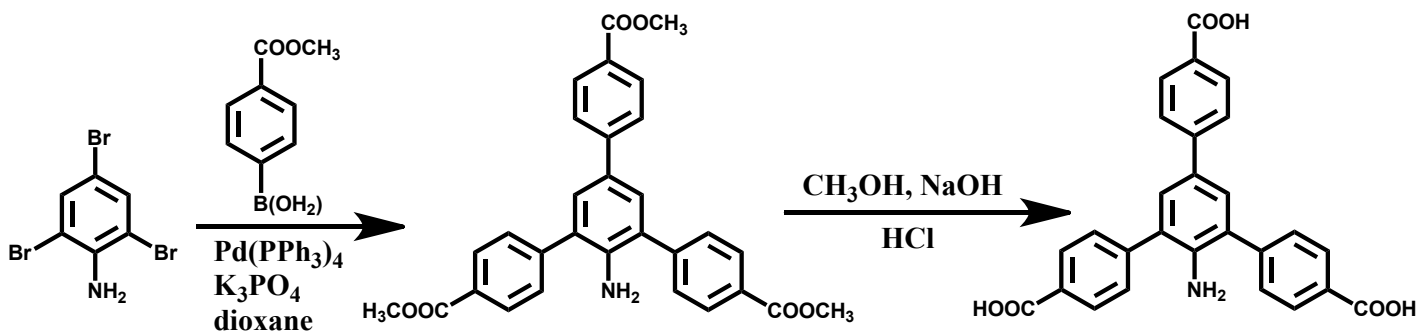

Scheme S1. Synthetic procedures of the $\mathrm{H}_{3} \mathrm{BTB}-\mathrm{NH}_{2}$ ligand.

\subsubsection{Dimethyl 2'-amino-5'-(4-(methoxycarbonyl)phenyl)-[1,1':3',1'-terphenyl]- 4,4"-dicarboxylate}

2,4,6-tribromoaniline (1.6 g, $4 \mathrm{mmol}$ ), Methyl 4-boronobenzoate (1.57 g, 14.4 $\mathrm{mmol}), \mathrm{Pd}\left(\mathrm{PPh}_{3}\right)_{4}(0.15 \mathrm{~g}, 0.13 \mathrm{mmol})$ and $\mathrm{K}_{3} \mathrm{PO}_{4}(3.82 \mathrm{~g}, 18.0 \mathrm{mmol})$ were placed in a $500 \mathrm{~mL}$ two-necked round bottom flask under a $\mathrm{N}_{2}$ gas atmosphere. The flask was further charged with a $200 \mathrm{~mL}$ of dry 1,4-dioxane, and the contents were heated for $48 \mathrm{~h}$. After the mixture was cooled to room temperature, the solvent was removed, and water was added. The water phase was washed with $\mathrm{CH}_{2} \mathrm{Cl}_{2}$. The mixed organic phases were dried with $\mathrm{MgSO}_{4}$. After the solvent was removed, the crude product was purified by column chromatography with $\mathrm{CH}_{2} \mathrm{Cl}_{2}$ as the eluent. ${ }^{1} \mathrm{H} \mathrm{NMR}$ (400 MHz, $\left.\mathrm{CDCl}_{3}\right) 3.87(\mathrm{~s}, 3 \mathrm{H}), 3.96(\mathrm{~s}, 6 \mathrm{H}), 7.59(\mathrm{~d}, 4 \mathrm{H}), 7.84(\mathrm{~s}, 2 \mathrm{H}), 8.16(\mathrm{~d}, 4 \mathrm{H})$. Anal. Calcd. for $\mathrm{C}_{24} \mathrm{H}_{21} \mathrm{NO}_{6}$ (mw 419): C, 68.73; N, 3.34; H, 5.05. Found: C, 68.80; N, $3.29 ; \mathrm{H}, 5.10$.
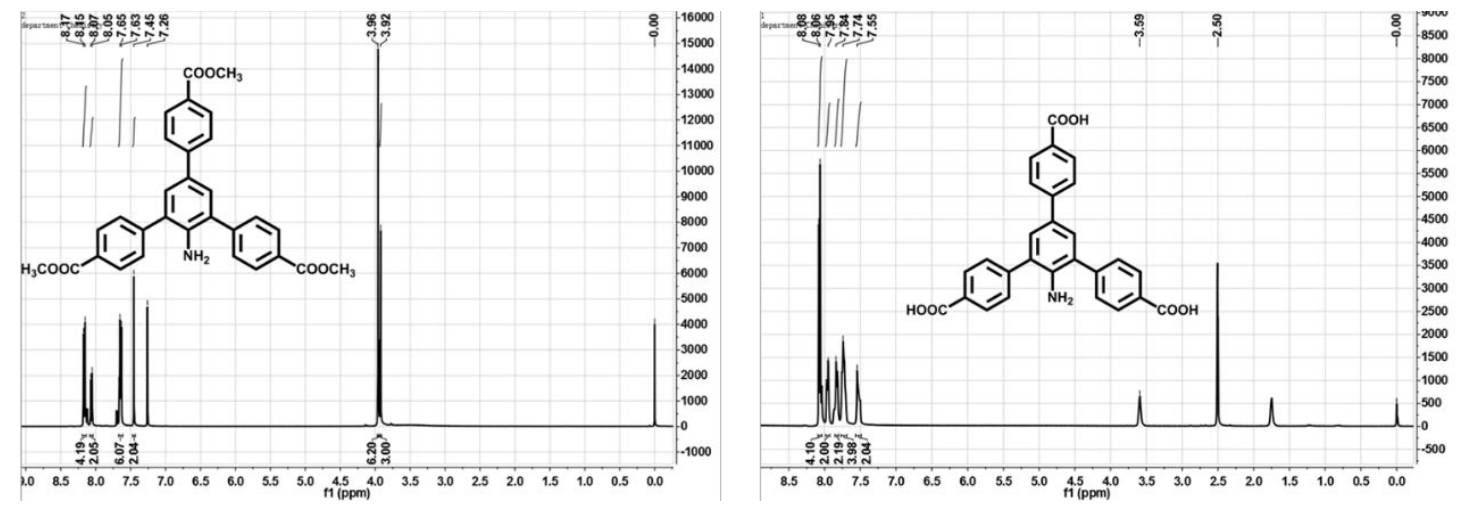

Figure S1. Nuclear magnetic resonance patterns of $\mathrm{H}_{3} \mathrm{BTB}-\mathrm{NH}_{2}$ ligand and its precursor. 


\subsubsection{2'-amino-5'-(4-carboxyphenyl)-[1,1':3',1'-terphenyl]-4,4"'-dicarboxylic acid}

Dimethyl 2'-amino-5'-(4-(methoxycarbonyl)phenyl)-[1,1':3',1"-terphenyl]-4,4"dicarboxylate $(2.0 \mathrm{~g}, 4.8 \mathrm{mmol})$ was dissolved in $50 \mathrm{~mL} \mathrm{MeOH}$, and then $50 \mathrm{~mL} 2 \mathrm{M}$ $\mathrm{NaOH}$ aqueous solution was added. The mixture was stirred at $50{ }^{\circ} \mathrm{C}$ overnight. The organic phase was removed, and the aqueous phase was acidified with diluted hydrochloric acid to give yellow precipitate, which was filtered and washed with water several times. ${ }^{1} \mathrm{H}$ NMR $\left(400 \mathrm{MHz}, \mathrm{CDCl}_{3}\right) 5.10(\mathrm{~s}, 2 \mathrm{H}), 7.62(\mathrm{~d}, 6 \mathrm{H}), 8.07(\mathrm{~d}$, $4 \mathrm{H}$ ), 12.78 (s, 3H). Anal. Calcd. for $\mathrm{C}_{21} \mathrm{H}_{15} \mathrm{NO}_{6}$ (mw 377): C, 66.84; N, 3.71; H, 4.01. Found: C, 66.78; N, 3.73; H, 4.00.

1.3 Synthesis of UPC-98. A mixture of $\mathrm{H}_{3} \mathrm{BTB}-\mathrm{NH}_{2}(0.008 \mathrm{~g}, 0.05 \mathrm{mmol})$ and $\mathrm{Zn}\left(\mathrm{NO}_{3}\right)_{2} \cdot 6 \mathrm{H}_{2} \mathrm{O}(0.060 \mathrm{~g}, 0.15 \mathrm{mmol})$ was dissolved in $1.00 \mathrm{~mL}$ DMF and $1.00 \mathrm{~mL}$ $\mathrm{H}_{2} \mathrm{O}$ and sealed in a $10 \mathrm{~mL}$ glass vial reactor. The vial was heated to $100{ }^{\circ} \mathrm{C}$ in 40 min, and maintained at $100{ }^{\circ} \mathrm{C}$ for $2400 \mathrm{~min}$. After slowly cooling down to ambient temperature at a speed of $0.1{ }^{\circ} \mathrm{C} / \mathrm{min}$, the crystals were collected and then rinsed with DMF. After dried at ambient condition, the light yellow block crystals were obtained with a yield of $84 \%$ based on Zinc. The elementary analysis calcd (\%) for UPC-98: C 60.3; H 3.04; N 2.61. Found: C 61.8; H 2.89; N 2.44. IR (KBr, $\left.\mathrm{cm}^{-1}\right): 3379$ (s), 1609 (s), 1377 (s), $1023(\mathrm{~m}), 1020(\mathrm{~m}), 868(\mathrm{w}), 773(\mathrm{~m}), 570(\mathrm{w})$.

1.4 Crystal structure determination. The Single crystal X-ray diffraction data were collected with the Agilent Technologies SuperNova diffractometer equipped with the graphite monochromatic $\mathrm{Cu} \mathrm{K \alpha}$ radiation. The structure was solved by means of the Superflip structure solution program employing the charge flipping method and refined by way of the ShelXL refinement package employing the least squares minimization method. The structure was anisotropically treated, while all hydrogen atoms were located in the calculated ideal positions and refined as riding on respective nonhydrogen atoms.

1.5 Gas sorption measurements. The activated samples were prepared by immersing the as-synthesized UPC-98 in the chromatographic grade $\mathrm{CH}_{3} \mathrm{OH}$ and $\mathrm{CH}_{2} \mathrm{Cl}_{2}$ to exchange solvent. After dried at ambient atmosphere, the samples were heated at 80 ${ }^{\circ} \mathrm{C}$ under vacuum for 5 hours. The gas adsorption experiments including $\mathrm{N}_{2}$ sorption at $77 \mathrm{~K}$ as well as the low-pressure $\mathrm{C}_{1}, \mathrm{C}_{2}$, and $\mathrm{C}_{3}$ light hydrocarbon sorption experiments at $273 \mathrm{~K}$ and $298 \mathrm{~K}$ were performed with the help of the ASAP-2020 surface area analyzer. The Brunauer-Emmett-Teller (BET) specific surface area and the corresponding pore size distribution were calculated based on $\mathrm{N}_{2}$ adsorption isotherm at $77 \mathrm{~K}$. To stabilize the temperature at 298,273 , and $77 \mathrm{~K}$, the water bath, ice water bath and liquid nitrogen bath were employed in the process of measurement, respectively.

1.6 Breakthrough experiments. The separation experiment of $\mathrm{C}_{2} \mathrm{H}_{2} / \mathrm{C}_{2} \mathrm{H}_{4}(1: 99)$ was completed employing a fixed bed. Before the experiment, the as-synthesized sample was heated at $80{ }^{\circ} \mathrm{C}$ for $12 \mathrm{~h}$ accompanied by the He flow $\left(2 \mathrm{~cm}^{3} \mathrm{~min}^{-1}\right)$ for activation. $90 \mathrm{mg}$ of activated crystal UPC-98 was packed into the stainless steel column as the adsorbent bed, and the gas mixture of $\mathrm{C}_{2} \mathrm{H}_{2} / \mathrm{C}_{2} \mathrm{H}_{4}\left(1: 99,2 \mathrm{~cm}^{3} \mathrm{~min}^{-1}\right)$ was led into the column at $298 \mathrm{~K}$. 
Section 2. Crystal data, structure and characterization of UPC-98

Table S1. Crystal data and structure refinement of UPC-98

\begin{tabular}{cc}
\hline Identification code & UPC-98 \\
\hline Empirical formula & $\mathrm{C}_{81} \mathrm{H}_{49} \mathrm{~N}_{3} \mathrm{O}_{18} \mathrm{Zn}_{4}$ \\
CCDC & 1912573 \\
Formula weight & 1613.71 \\
Temperature/K & $293(2)$ \\
Crystal system & monoclinic \\
Space group & $\mathrm{C}_{2} / \mathrm{c}$ \\
$\mathrm{a} / \AA$ & $29.1163(3)$ \\
$\mathrm{b} / \AA$ & $16.3503(2)$ \\
$\mathrm{c} / \AA$ & $42.8625(6)$ \\
$\alpha /{ }^{\circ}$ & 90 \\
$\beta /{ }^{\circ}$ & $96.7493(12)$ \\
$\gamma /{ }^{\circ}$ & 90 \\
Volume/ $\AA^{3}$ & $20263.6(4)$ \\
$\mathrm{Z}$ & 8 \\
$\mathrm{D}_{\mathrm{c}} / \mathrm{g}$ cm & -3 \\
$\mu / \mathrm{mm}^{-1}$ & 1.058 \\
$\mathrm{~F}(000)$ & 1.519 \\
$2 \Theta$ range for data & 6560.0 \\
Reflections collected & 9.724 to 141.604 \\
Independent reflections & 28260 \\
Data/restraints/parameters & $14679\left[\mathrm{R}_{\text {int }}=0.0216, \mathrm{R}_{\text {sigma }}=0.0336\right]$ \\
Goodness-of-fit on $\mathrm{F}^{2}$ & $14679 / 0 / 956$ \\
Final $\mathrm{R}$ indexes $[\mathrm{I}>=2 \sigma$ & 1.099 \\
Final $\mathrm{R}$ indexes [all data $]$ & $\mathrm{R}_{1}=0.1011, \mathrm{wR}_{2}=0.2540$ \\
Largest diff. peak/hole $/ \mathrm{e}$ & $0.1219, \mathrm{wR}_{2}=0.2815$ \\
\hline
\end{tabular}

Table S2. Selected bond lengths $(\AA)$ for UPC-98.

\begin{tabular}{cccccc}
\hline Atom & Atom & Length $/ \AA$ & Atom & Atom & Length/ $\AA$ \\
\hline $\mathrm{Zn} 1$ & $\mathrm{O} 3$ & $1.940(5)$ & $\mathrm{Zn} 3$ & $\mathrm{~N} 16$ & $2.080(7)$ \\
$\mathrm{Zn} 1$ & $\mathrm{O} 4$ & $1.928(4)$ & $\mathrm{Zn} 3$ & $\mathrm{O} 11^{3}$ & $1.959(5)$ \\
$\mathrm{Zn} 1$ & $\mathrm{O} 1^{1}$ & $1.943(4)$ & $\mathrm{Zn} 3$ & $\mathrm{O} 14^{4}$ & $1.914(6)$ \\
$\mathrm{Zn} 1$ & $\mathrm{O}^{2}$ & $1.935(4)$ & $\mathrm{Zn} 4$ & $\mathrm{~N} 38$ & $2.213(3)$ \\
$\mathrm{Zn} 2$ & $\mathrm{~N} 27$ & $2.056(5)$ & $\mathrm{Zn} 4$ & $\mathrm{O} 13^{5}$ & $1.932(6)$ \\
$\mathrm{Zn} 2$ & $\mathrm{O} 2^{1}$ & $1.910(4)$ & $\mathrm{Zn} 4$ & $\mathrm{O}^{6}$ & $1.920(3)$ \\
$\mathrm{Zn} 2$ & $\mathrm{O}^{2}$ & $1.960(4)$ & $\mathrm{N} 16$ & $\mathrm{C} 31$ & $1.443(7)$ \\
$\mathrm{Zn} 3$ & $\mathrm{O} 12$ & $1.941(5)$ & $\mathrm{O} 3$ & $\mathrm{C} 2$ & $1.257(7)$ \\
\hline
\end{tabular}

UPC-98: ${ }^{1}-1 / 2+X,-1 / 2+Y,+Z ;{ }^{2}+X,-1+Y,+Z ;{ }^{3} 1 / 2+X, 1 / 2+Y,+Z ;{ }^{4}+X, 1+Y,+Z ;{ }^{5} 1-X,-Y, 1-Z ;{ }^{6} 1 / 2-X,-1 / 2-Y, 1-Z$ 


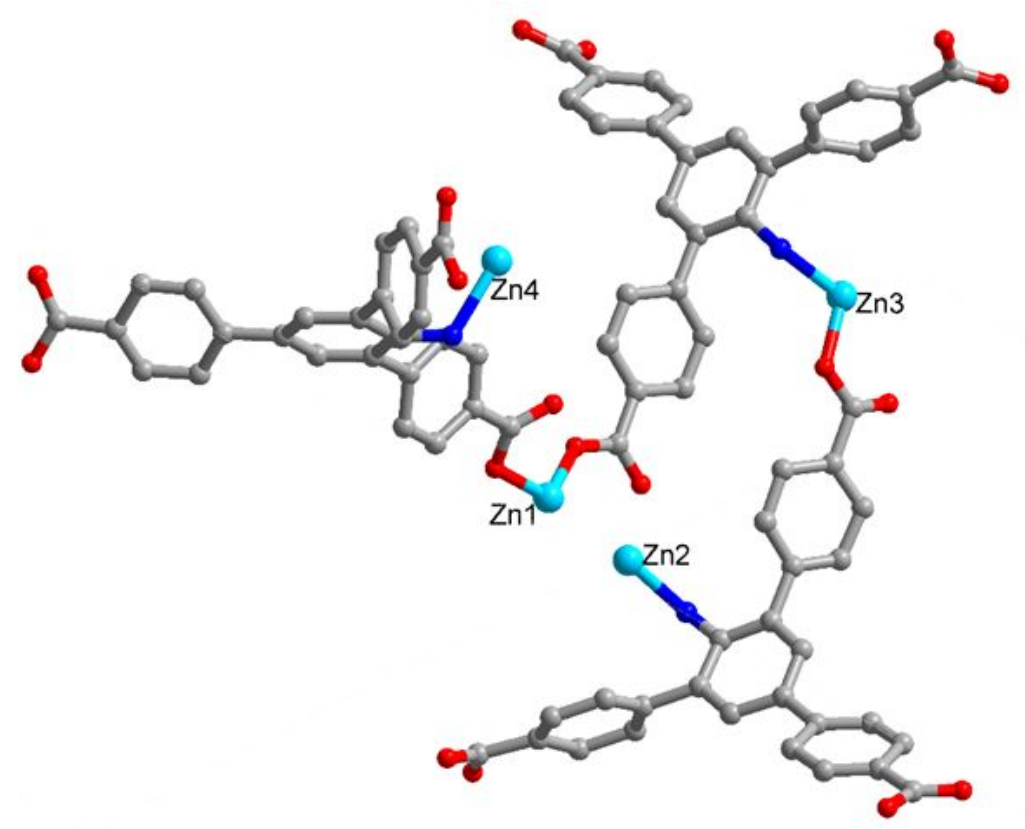

Figure S2. The asymmetric structural unit of UPC-98.

(a)

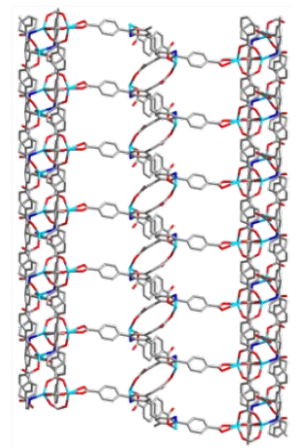

(b)

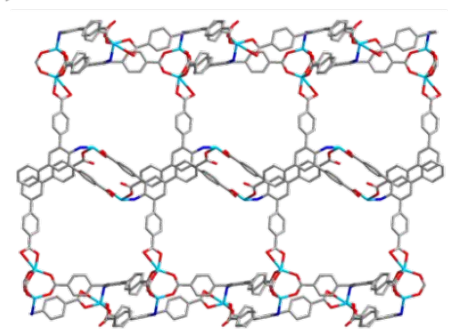

(c)

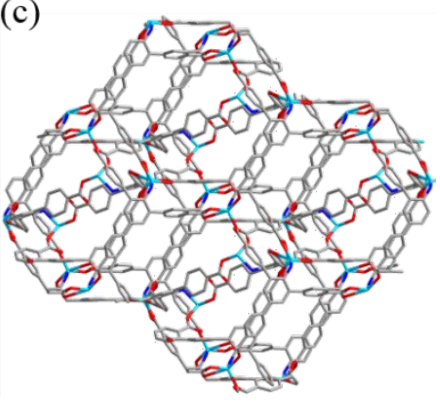

Figure S3. The three-dimensional pileup of the basic interpenetrating in UPC-98 along $a, b$ and $c$ axes.

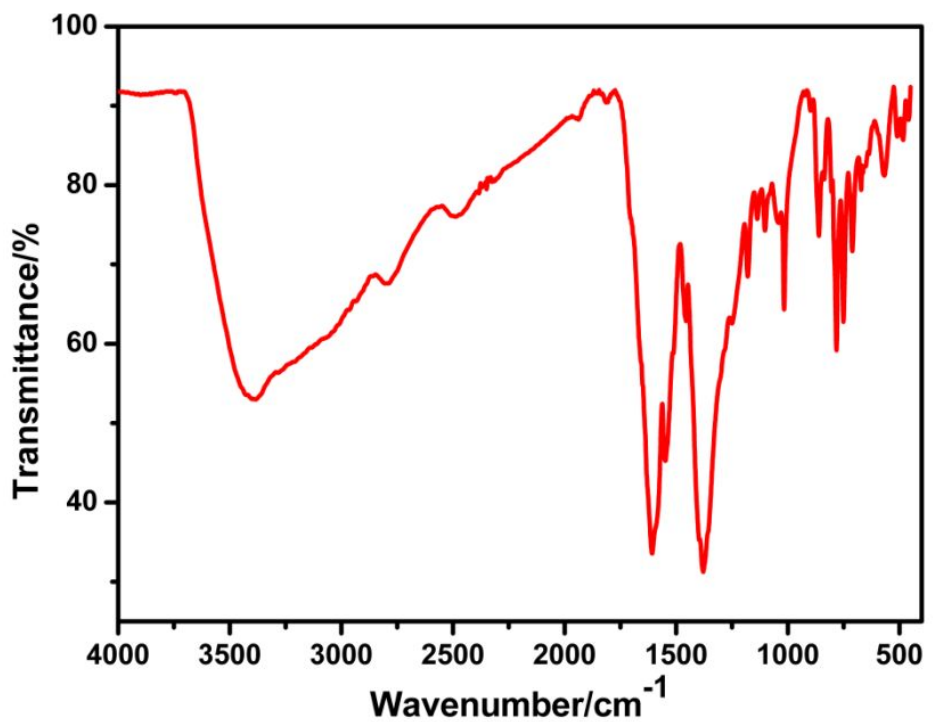

Figure S4. The IR of UPC-98. 


\section{Section 3. Simulation and calculation for gas selectivity by IAST methods}
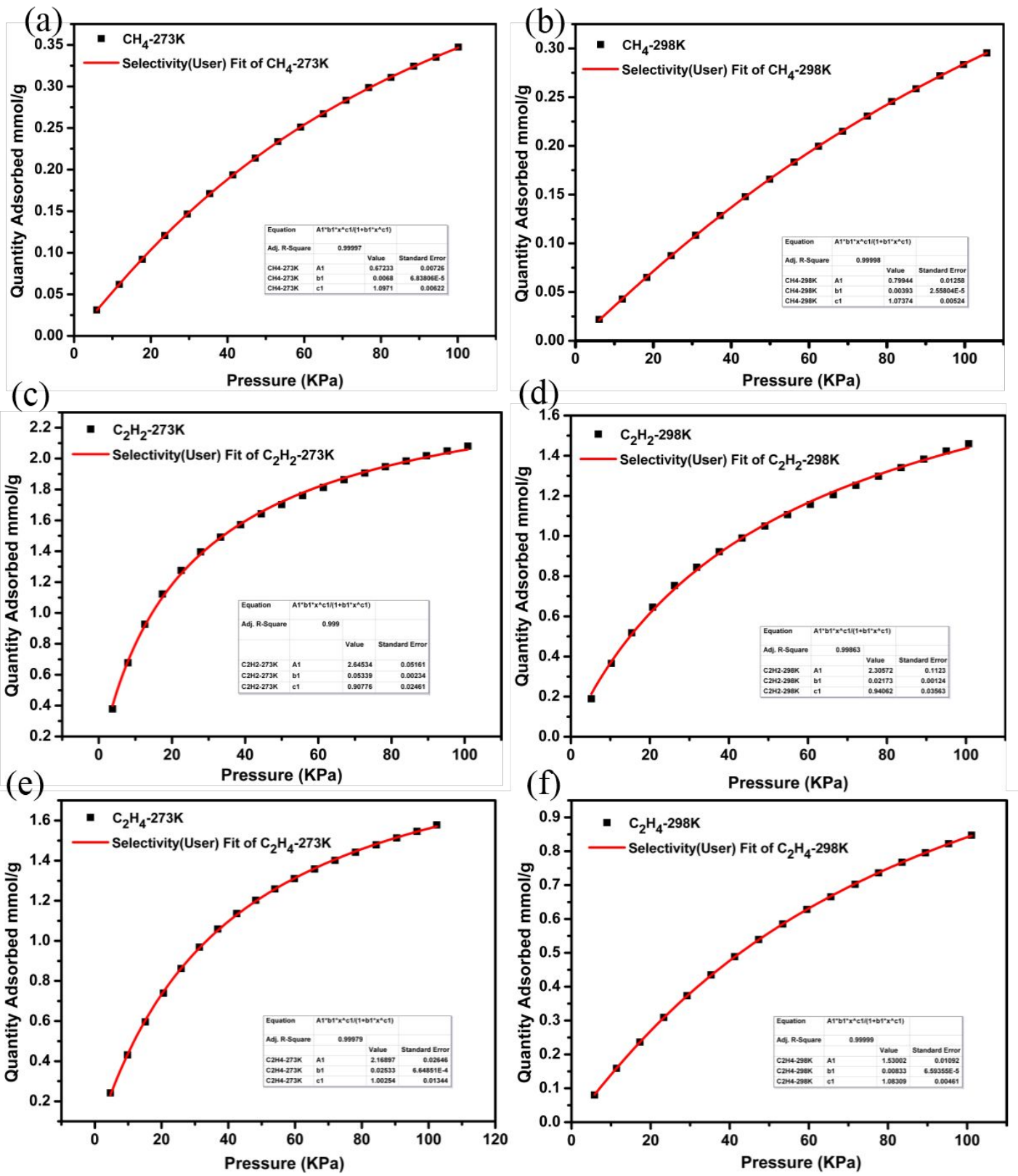

Figure S5. The detail of virial equation (solid lines) fitting to the experimental gases adsorption data at $273 \mathrm{~K}$ and $298 \mathrm{~K}$ for UPC-98 by IAST methods: $\mathrm{CH}_{4}(\mathrm{a}, \mathrm{b}) ; \mathrm{C}_{2} \mathrm{H}_{2}$ (c, d); $\mathrm{C}_{2} \mathrm{H}_{4}(\mathrm{e}, \mathrm{f})$. 
(a) 1.6

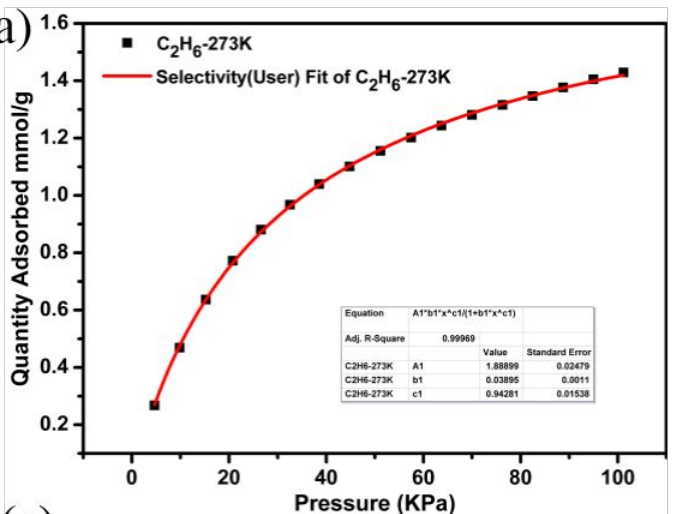

(c)

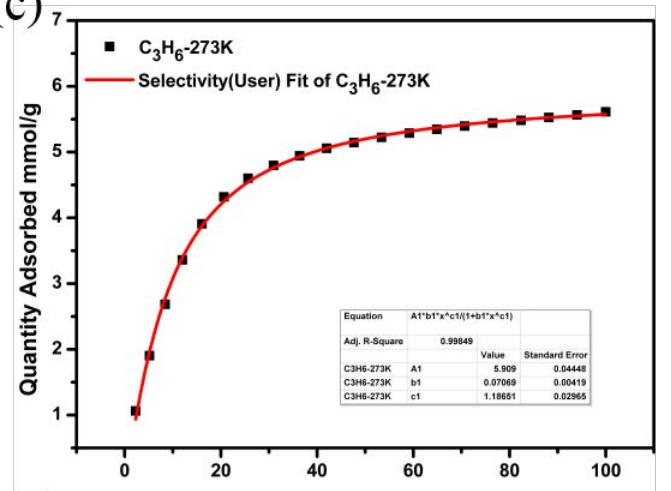

(e)

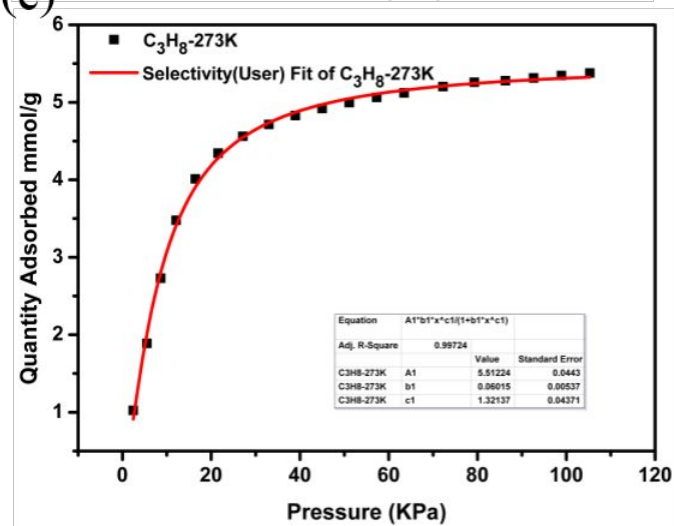

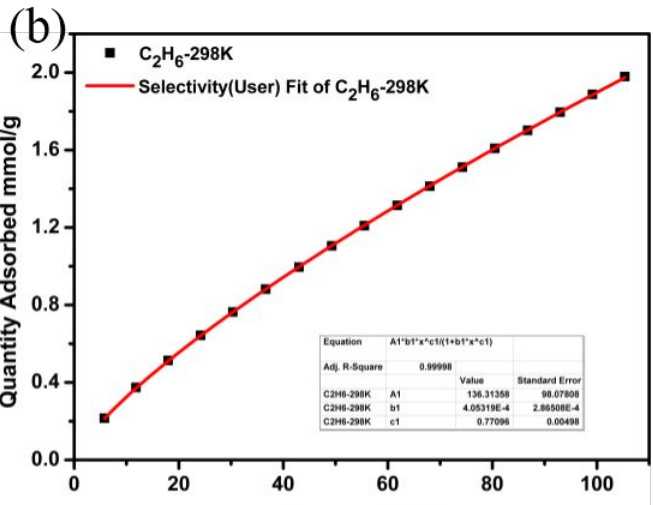

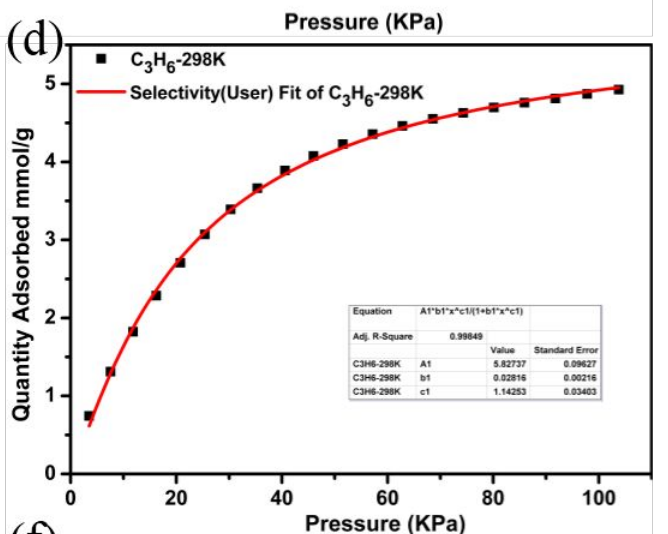

(f)

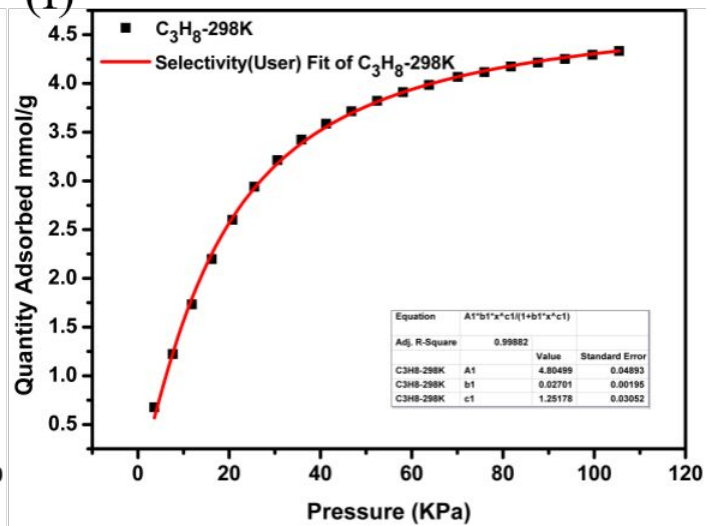

Figure S6. The detail of virial equation (solid lines) fitting to the experimental gases adsorption data at $273 \mathrm{~K}$ and $298 \mathrm{~K}$ for UPC-98 by IAST methods: $\mathrm{C}_{2} \mathrm{H}_{6}(\mathrm{a}, \mathrm{b}) ; \mathrm{C}_{3} \mathrm{H}_{6}$ (c, d); $\mathrm{C}_{6} \mathrm{H}_{8}(\mathrm{e}, \mathrm{f})$. 


\section{Section 4. Simulation and calculation for $Q_{\text {st }}$ by Clausius-Clapeyron equation}

(a)

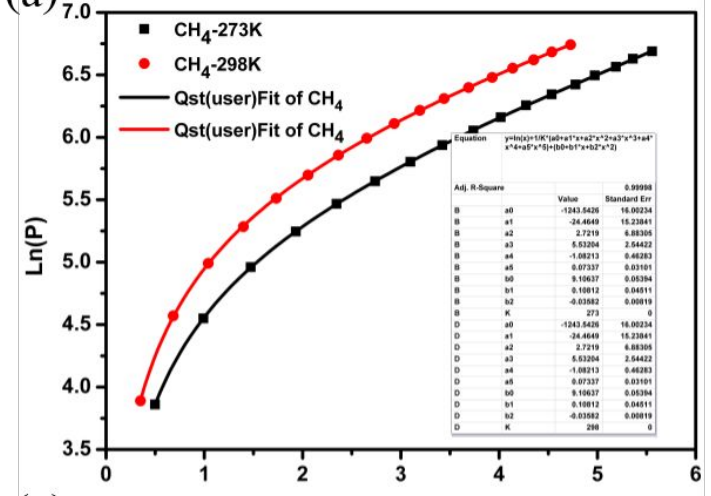

(c)

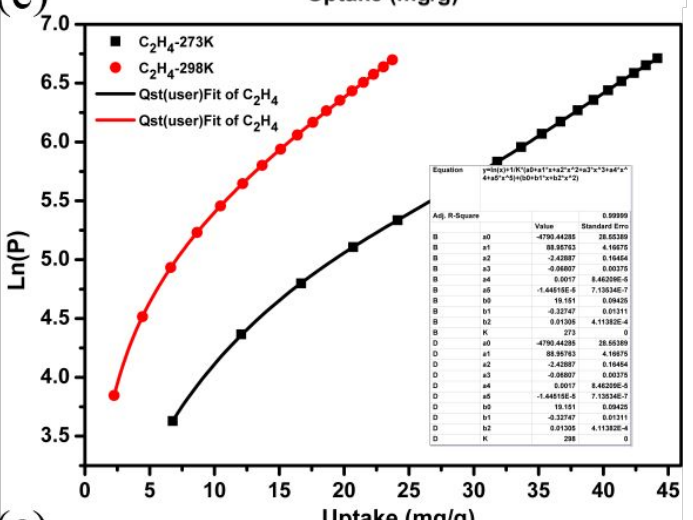

(e)

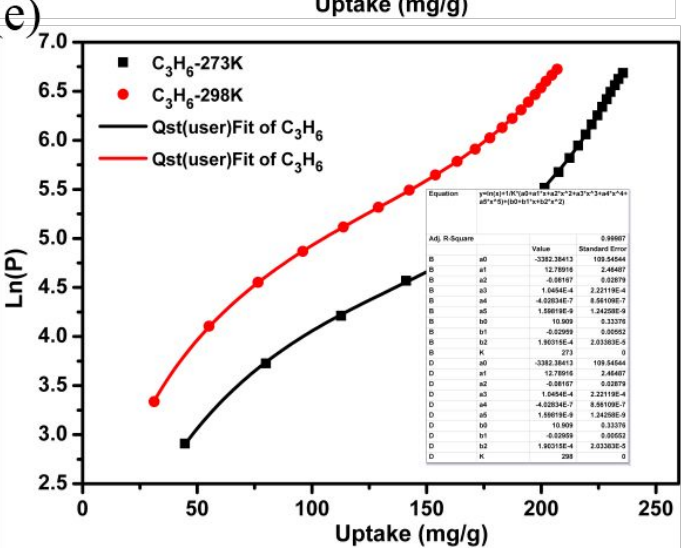

(b)

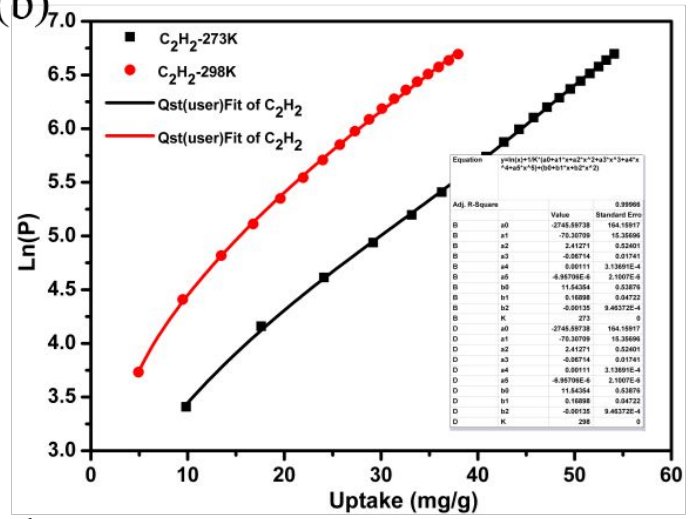

$(\mathrm{d})_{7}$

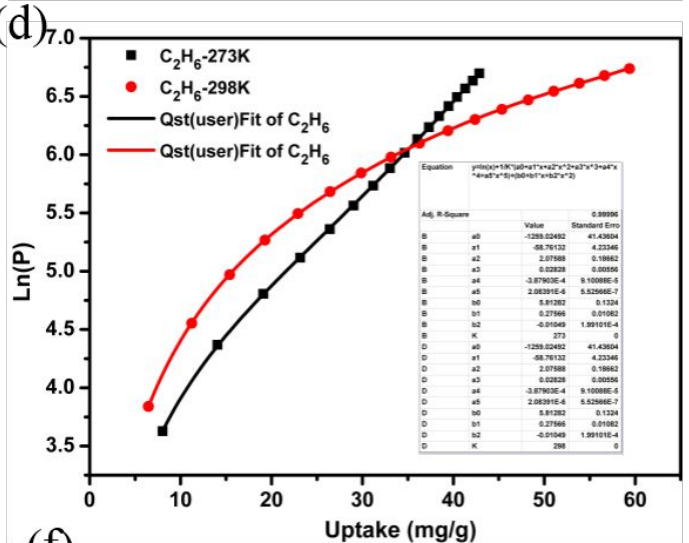

(f)

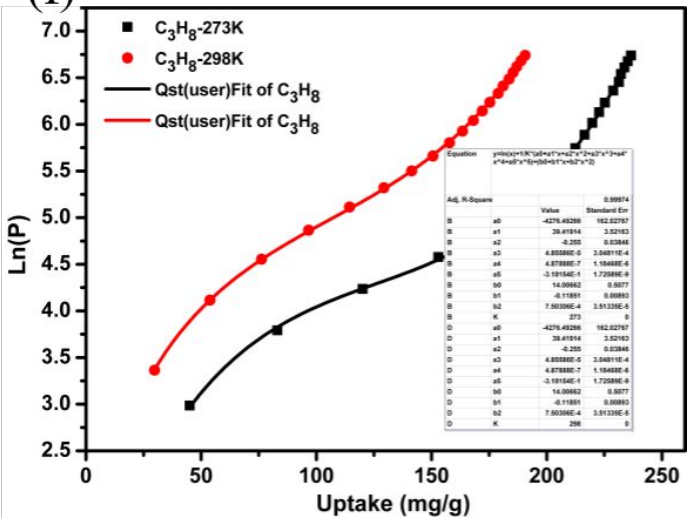

Figure S7. The parameters and optimized adsorption isotherms for calculated $Q_{\text {st }}$ of $\mathrm{CH}_{4}-\mathrm{C}_{3} \mathrm{H}_{8}$ using a variant of the Clausius-Clapeyron equation: $\mathrm{CH}_{4}$ (a); $\mathrm{C}_{2} \mathrm{H}_{2}$ (b); $\mathrm{C}_{2} \mathrm{H}_{4}(\mathrm{c}) ; \mathrm{C}_{2} \mathrm{H}_{6}(\mathrm{~d}) ; \mathrm{C}_{3} \mathrm{H}_{6}(\mathrm{e}) ; \mathrm{C}_{3} \mathrm{H}_{8}(\mathrm{f})$. 
Table S3. Light hydrocarbon adsorption data $\left(\mathrm{cm}^{3} \cdot \mathrm{g}^{-1}\right)$ for selected MOFs.

\begin{tabular}{|c|c|c|c|c|c|c|c|c|}
\hline MOFs & $\mathrm{T} / \mathrm{K}$ & $\mathrm{CH}_{4}$ & $\mathrm{C}_{2} \mathrm{H}_{2}$ & $\mathrm{C}_{2} \mathrm{H}_{4}$ & $\mathrm{C}_{2} \mathrm{H}_{6}$ & $\mathrm{C}_{3} \mathrm{H}_{6}$ & $\mathrm{C}_{3} \mathrm{H}_{8}$ & ref \\
\hline \multirow[t]{2}{*}{ UPC-98 } & 273 & 8.1 & 43.1 & 31.3 & 29.5 & 120.5 & 118.4 & This work \\
\hline & 298 & 5.9 & 30.2 & 17.6 & 45.4 & 116.5 & 97.4 & \\
\hline \multirow[t]{2}{*}{ UPC-33 } & 273 & 9.7 & 65.1 & 43.6 & 51.8 & 114.2 & 111.8 & 1 \\
\hline & 298 & 7.0 & 44.3 & 31.1 & 35.0 & 94.3 & 93.6 & \\
\hline \multirow[t]{2}{*}{ FJI-C1 } & 273 & & 135.9 & 85.2 & 123.6 & & 160.9 & 2 \\
\hline & 298 & 9.7 & 93.8 & 64.0 & 87.4 & & 141.9 & \\
\hline \multirow[t]{2}{*}{ FJI-C4 } & 273 & 32.7 & 82.8 & 70.1 & 73.4 & & 74.7 & 3 \\
\hline & 298 & 18.4 & 72.5 & 61.4 & 66.3 & & 71.5 & \\
\hline UTSA-33a & 296 & $9.2 \mathrm{mg} / \mathrm{g}$ & 97.1 & 76.2 & 83.0 & & & 4 \\
\hline \multirow[t]{2}{*}{ 1-mim } & 273 & 14.65 & 119.42 & 92.37 & 101.03 & & 102.92 & 5 \\
\hline & 297 & 10.64 & 76.26 & 64.95 & 79.91 & & 96.87 & \\
\hline \multirow[t]{2}{*}{ 1-eim } & 273 & 19.32 & 117.84 & 87.30 & 99.348 & & 97.36 & 5 \\
\hline & 297 & 11.48 & 73.70 & 61.29 & 75.38 & & 86.60 & \\
\hline \multirow[t]{2}{*}{ 1-pim } & 273 & 16.24 & 101.42 & 84.54 & 93.78 & & 97.31 & 5 \\
\hline & 297 & 9.70 & 65.00 & 53.72 & 71.65 & & 83.29 & \\
\hline \multirow[t]{2}{*}{ 1-buim } & 273 & 14.08 & 93.54 & 73.16 & 81.77 & & 83.46 & 5 \\
\hline & 297 & 8.86 & 56.14 & 48.70 & 63.00 & & 75.22 & \\
\hline \multirow[t]{3}{*}{ M'MOF-20 } & 195 & 70 & 268 & 81 & 77 & & 39 & 6 \\
\hline & 273 & & 95.0 & 53.0 & & & 43.0 & \\
\hline & 298 & 8.0 & 81.0 & 44.0 & 49.0 & & 47.0 & \\
\hline \multirow[t]{2}{*}{ UPC-35 } & 273 & 11.0 & 72.5 & 56.4 & 70.1 & 138.1 & 128.7 & 7 \\
\hline & 298 & 4.8 & 44.4 & 35.9 & 40.9 & 118.3 & 111.3 & \\
\hline
\end{tabular}




\section{References}

1. W. D. Fan, Y. T. Wang, Q. Zhang, A. Kirchon, Z. Y. Xiao, L. L. Zhang, F. N. Dai, R. M. Wang, D. F. Sun, Chem. Eur. J. 2017, 23, 1-8.

2. Y. B. Huang, Z. J. Lin, H. R. Fu, F. Wang, M. Shen, X. S. Wang, R. Cao, ChemSusChem. 2014, 7, 2647-2653.

3. L. Li, X. S. Wang, J. Liang, Y. B. Huang, H. F. Li, Z. J. Lin, R. Cao, ACS Appl. Mater. Interfaces. 2016, 8, 9777-9781.

4. Y. B. He, Z. J. Zhang, S. C. Xiang, F. R. Fronczek, R. Krishna, B. L. Chen, Chem. Eur. J. 2012, 18, 613-619.

5. H. R. Fu, J. Zhang, Inorg. Chem. 2016, 55, 3928-3932.

6. N. Sikdar, S. Bonakala, R. Haldar, S. Balasubramanian, T. K. Maji, Chem. Eur. J. 2016, 22, 6059-6070.

7. Y. T. Wang, W. D. Fan, X. Wang, Y. F. Han, L. L. Zhang, D. Liu, F. N. Dai, D. F. Sun, Inorg, Chem. Front., 2018, 5, 2408-2412. 